\title{
Listeria spondylodiscitis: an uncommon etiology of a common condition; a case report
}

\author{
Rand Al Ohaly ${ }^{*^{*}}$, Nischal Ranganath ${ }^{1}$, Medina G. Saffie ${ }^{2}$ and Anjali Shroff ${ }^{1}$
}

\begin{abstract}
Background: Listeriosis is a severe food-borne infection caused by the Gram-positive rod, Listeria monocytogenes. Despite the low incidence (3-8 cases per million), Listeriosis has a case fatality rate of 20-30\% as it occurs predominantly in immunocompromised individuals at extremes of age, diabetics and pregnant women. Listeriosis classically presents as a febrile gastroenteritis, isolated bacteremia, meningitis, or maternal-fetal infections. Focal bone and joint infection are rare and primarily involve orthopedic implant devices. Here, we present the first case of Listeria-associated spondylodiscitis.

Case presentation: A 79-year-old male presents with acute-on-chronic back pain in the absence of risk factors or exposures, aside from age. On radiological imaging, spondylodiscitis of L3-L4 was diagnosed. Subsequently, a CTguided biopsy was performed to aid in confirming microbiological aetiology. Listeria monocytogenes was grown in culture and patient received appropriate antibacterial therapy.

Conclusion: The case highlights the utility of image-guided tissue sampling in aiding diagnosis and management in patients with vertebral osteomyelitis. It also encourages consideration of uncommon organisms such as Listeria as an etiology of vertebral osteomyelitis, even in the absence of prosthetic implants.
\end{abstract}

Keywords: Listeria, Spondylodiscitis, Bone \& joint infection, Listeriosis, Case report

\section{Introduction}

L. monocytogenes is a gram-positive, facultative, intracellular bacillus responsible for the febrile gastrointestinal illness, Listeriosis, acquired from contaminated food that typically occurs sporadically or in outbreaks [1]. There are several serotypes of Listeria distinguished based on the cell-surface $\mathrm{O}$ and $\mathrm{H}$ antigens, with the three serotypes $1 / 2 c, 1 / 2 b$, and $4 b$ accounting for greater than $95 \%$ of human illnesses [2]. The organism possesses several features including an intrinsic resistance to high acidity and salinity, as well as the capacity to grow at low temperatures that support it's efficacy in foodborne transmission [3]. Following ingestion, systemic infection can be established through tissue penetration across the gastrointestinal tract or hematogenous spread to sterile sites including the central nervous system (CNS), heart, liver, spleen, and placenta [3]. Consistent with this, $L$. monocytogenes associated bacteremia, meningoencephalitis, infective endocarditis, and endovascular graft infections have been previously reported [4-7]. Interestingly, however, this organism rarely causes bone and joint infections, and if so, occurs typically in the context of prosthetic material providing a site for bacterial seeding. Herein, we present the first case of Spondylodiscitis in an individual void of known risk factors.

\footnotetext{
* Correspondence: rand.alohaly@medportal.ca

'Department of Medicine, McMaster University, Hamilton, ON, Canada

Full list of author information is available at the end of the article
}

C C The Author(s). 2020 Open Access This article is licensed under a Creative Commons Attribution 4.0 International License, which permits use, sharing, adaptation, distribution and reproduction in any medium or format, as long as you give appropriate credit to the original author(s) and the source, provide a link to the Creative Commons licence, and indicate if changes were made. The images or other third party material in this article are included in the article's Creative Commons licence, unless indicated otherwise in a credit line to the material. If material is not included in the article's Creative Commons licence and your intended use is not permitted by statutory regulation or exceeds the permitted use, you will need to obtain permission directly from the copyright holder. To view a copy of this licence, visit http://creativecommons.org/licenses/by/4.0/ The Creative Commons Public Domain Dedication waiver (http://creativecommons.org/publicdomain/zero/1.0/) applies to the data made available in this article, unless otherwise stated in a credit line to the data. 


\section{Case presentation}

A 79-year-old gentleman presented with a 3-week history of acute, progressively worsening right-sided back pain radiating to the right groin and anterior thigh. He was admitted due to reduced mobility secondary to pain and an inability to safely function independently at home. A risk assessment for red flag symptoms including spinal cord or cauda equina compression, progressive neurological deficits, new urinary retention, faecal incontinence, and constitutional symptoms were absent. He was in his usual state of health prior to the onset of these symptoms. He denied any history of fevers, rigors, gastrointestinal symptoms, inciting trauma, or recent dental or surgical procedures. Social history included a 30-pack-year smoking history, minimal alcohol use, and denied intravenous substance use. His past medical history included hypertension, dyslipidaemia, gout and reflux. His past surgical history involved bilateral hernia repair with mesh, bilateral subclavian to carotid artery bypass and repair of an endovascular infrarenal abdominal aortic aneurysm, which required embolization for a leak at the left internal iliac artery 4 years later. Home medications included Clopidogrel, Hydrochlorothiazide, Perindopril, Allopurinol, Pantoprazole, Rosuvastatin, and Tamsulosin. There was no history of immunosuppression or use of immune modulating medications, particularly glucocorticoids. He denied any recent travel, animal exposures, consumption of processed meats, cheeses or other dairy products.

The patient was afebrile and hemodynamically stable at presentation and throughout his hospital stay. Physical examination of the cardiorespiratory, abdominal, and neurological systems was within normal limits. A focused examination revealed no tenderness on spinal palpation, a negative straight leg raise test, no pain on leg roll, and a normal gait. Relevant laboratory results included lymphopenia $\left(0.7 \times 10^{9} / \mathrm{L}\right)$, anaemia (haemoglobin $101 \mathrm{~g} / \mathrm{L}$ ), LDH of $202 \mathrm{U} / \mathrm{L}$, and CRP $34.9 \mathrm{mg} / \mathrm{L}$, which later increased to $87.9 \mathrm{mg} / \mathrm{L}$. Multiple sets of blood cultures were repeatedly negative. During his admission, he had an episode of selfresolving diarrhea. Interestingly, despite no prior antibiotic exposure, a stool sample was positive for $C$. difficile toxin by PCR. This, however, was more representative of a colonisation rather than a true infection.

Radiographs of the hip and pelvis showed multi-level degenerative disc disease without evidence of fracture. CT Chest/Abdomen/Pelvis with endovascular protocol was negative for endoleak or dissection, but incidentally demonstrated interval L3-L4 discitis with destruction of associated endplates. Transthoracic echocardiogram effectively ruled out the presence of valve disease or vegetations. An MRI with gadolinium was subsequently performed and confirmed the diagnosis of L3-L4 discitis and osteomyelitis (Figs. 1, 2). Given the clinical stability

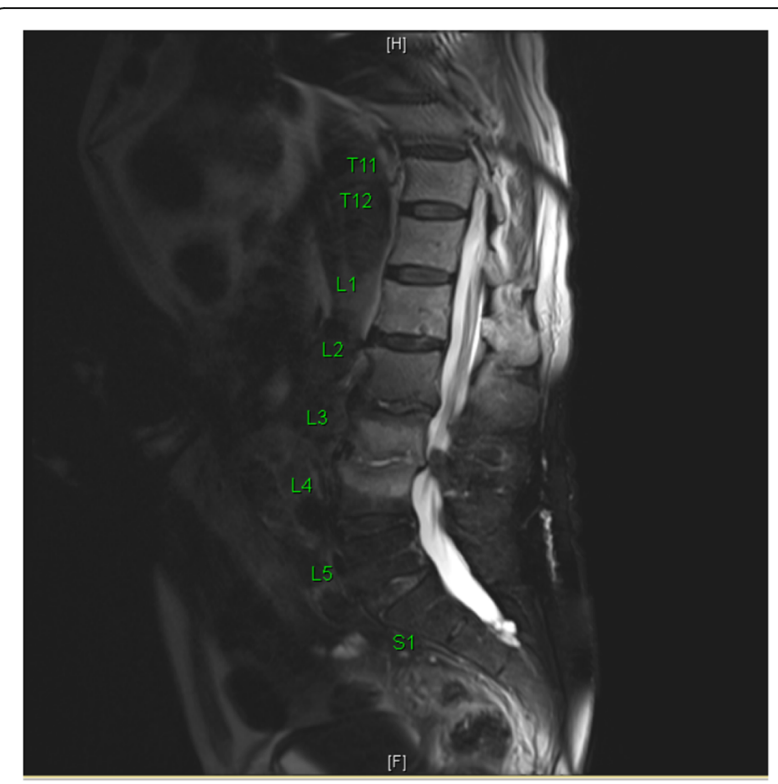

Fig. 1 Sagittal T2 Fat Sat: high signal intensity within the L3-L4 disC space involving the inferior endplate of $L 3$ and superior end plate of L4 with end plate irregularity

and a lack of clear causative pathogen on blood culture, antibiotic administration was delayed to accommodate a fluoroscopy-guided percutaneous aspiration and biopsy. Fluid aspirate returned positive for Listeria monocytogenes in broth culture. Fungal and mycobacterial cultures remained negative. L. monocytogenes was thus determined to be the aetiology and the patient was

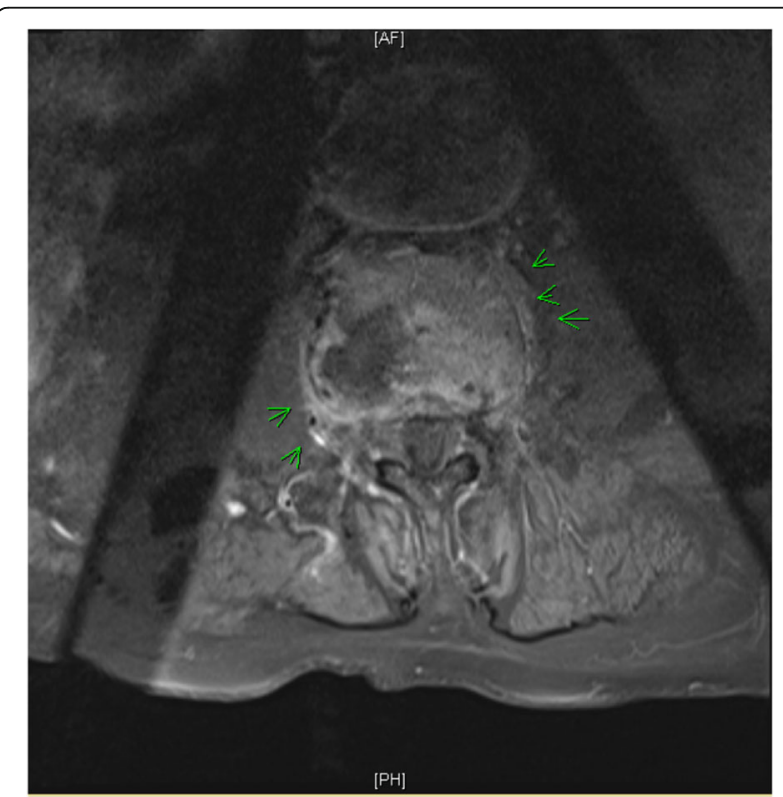

Fig. 2 Axial T1 post-gadolinium: (no pre-GAD) images. Small amount of enhancing paravertebral soft tissue. No evidence to suggest paravertebral or epidural abscess formation 
started on a 6-week course of Ampicillin, with rapid clinical improvement and normalization of CRP on serial assessment. The patient was subsequently seen in follow-up with both the Infectious Disease and Vascular Surgery services at 2- and 6-month time points with ongoing clinical recovery and no recurrence of disease.

\section{Discussion and conclusions}

Despite its rarity (between 3 to 8 cases per million worldwide), the average case-fatality rate of invasive Listeriosis is up to $20-30 \%$, even with appropriate antimicrobial therapy $[8,9]$. This is largely attributable to a predilection for immunocompromised individuals with extremes of age (neonates and elderly age $\geq 65$ years), diabetics and pregnant women. Additional high-risk populations include those with hematologic malignancy, end-stage renal disease, AIDS, organ transplantation, iron-overload states, alcoholism and other chronic liver diseases $[3,8]$. In particular, the risk of invasive Listeriosis is noted with the use of TNF- $\alpha$ inhibitors as it suppresses T-cell mediated immunity; the primary defence against this organism [10-12].

Listeriosis classically presents as febrile gastroenteritis, isolated bacteremia, meningitis, encephalitis, or maternalfetal infections $[2,8]$. Localized CNS involvement often presents as subcortical brain or intramedullary spinal abscesses [13-16]. Interestingly, bone and joint infection secondary to Listeria is uncommon and primarily involves orthopedic implant devices including prosthetic joints [11]. In the French case series of Listeria-associated bone and joint infections, $84 \%$ of infections involved prosthetic material, with a minority of cases involving native joint infections [11]. To the best of our knowledge, there have only been 2 prior cases of Listeria-associated vertebral osteomyelitis (OM) occurring in the context of risk factors including spine surgery and immunosuppression, as well as 1 case of Listeria bacteremia with spondylodiscitis in the absence of clear risk factors $[13,14,17]$.

Here we present the first case of Listeria-associated spondylodiscitis in a 79-year-old male in the absence of significant risk factors, bacteremia, comorbidities (including prostheses), or exposures. The patient's advanced age is the only shared risk factor with the previously published case reports. Given the non-specific presentation of back pain and absence of infectious prodrome, the finding of vertebral spondylodiscitis was made incidentally. Further investigation of the spondylodiscitis yielded repeated negative blood cultures; thus, necessitating a CT-guided biopsy and aspiration for diagnosis.

\section{Diagnostic yield of biopsy}

The determination of a microbiologic diagnosis, as in this case, can be challenging in the absence of obvious exposures, negative blood cultures, and serology. In these circumstances, fluoroscopy-guided diagnostic aspiration biopsy is valuable in obtaining samples for microbiologic testing and in providing diagnostic clarity between infectious, malignant or degenerative processes [18]. CT-guided biopsy has variable sensitivity in determining infectious etiology ranging from 30.4 to $74 \%$ [18-20], with a recent metanalysis demonstrating $52.2 \%$ (95\% CI, 45.8-58.5\%) sensitivity and 99.9\% (95\% CI, 94.5-100\%) specificity [21]. This strategy therefore represents an excellent modality to obtain a microbiologic diagnosis; but the absence of a positive culture does not necessarily rule out infection. From a patient outcome standpoint, CT-guided aspiration for culture also prevents the need for open surgical intervention and biopsy in $50-60 \%$ of cases [18] and can facilitate targeted antibiotic therapy [22].

\section{Timing of antibiotics in relation to diagnostic sampling}

As in this case, empiric therapy should be delayed, if the patient is haemodynamically stable with no neurological compromise, in order to obtain tissue samples for culture as per the IDSA guidelines for native vertebral osteomyelitis management $[13,17]$. The postponement of antibiotic therapy does improve microbiological yield and can be deferred in the absence of life-threatening conditions or spinal cord compromise [23]. However, initiation of antibiotics does not preclude undertaking a biopsy, as a recent metanalysis demonstrated a nonsignificant difference in biopsy yield of $32 \%$ vs $43 \%$ in those with and without prior antibiotic exposure [24]. In cases where antibiotic therapy has already been started, it was demonstrated that interrupting and withholding antibiotics for approximately 14 days pre-CT guided tissue biopsy had a better yield (76.9\%) when compared to holding for only 3 days pre-biopsy (41.2\%) [25]. This can vary, however, depending on the pharmacokinetics, dose and duration, and bone penetration of the selected empiric antibiotic. Nevertheless, a short duration of empiric antibiotic exposure does not negatively impact pathogen recovery and is therefore not a contraindication for vertebral biopsy [26]. If image-guided sampling of tissue is not feasible or available, or signs of clinical instability or focal neurological involvement is identified, appropriate empiric therapy and timely consultation of the Infectious Diseases and Spinal Surgery services is warranted [18].

\section{Conclusion}

In summary, this case report presents the first case of Listeria-associated spondylodiscitis in the absence of a clear exposure, wherein the only risk factor was age. Consideration for Listeria-associated osteomyelitis should be a part of the differential diagnosis, even in the absence of prosthetic material. This is particularly important in 
those with appropriate epidemiologic risk factors, but should also be considered in those living in areas with relatively low incidences of Listeria infection as in this case. Lastly, the present case strongly supports the utility of tissue sampling in all cases of suspected OM, that have no ongoing neurological or hemodynamic compromise, in order to achieve microbiological etiology. This can facilitate the use of the narrowest spectrum and most effective antibiotic appropriate for optimal patient care.

\section{Abbreviations}

CNS: Central nervous system; OM: Osteomyelitis

\section{Acknowledgements}

We kindly thank the patient involved in the case report for allowing us to share the case and associated discussion in further advancing scientific discourse.

\section{Authors' contributions}

R.A.O and N.R. contributed equally to the design, analysis and interpretation of clinical data, and writing the manuscript. A.S. and M.G.S. conceived and designed the study, contributed to data analysis and interpretation, as well as drafting and editing the manuscript. All authors read and approved the final manuscript.

\section{Funding}

There are no funding sources to declare.

\section{Availability of data and materials}

Not applicable.

\section{Ethics approval and consent to participate}

Not applicable.

\section{Consent for publication}

Consent was taken from the patient before starting the case report, and signature was acquired, allowing use of deidentified data and images.

\section{Competing interests}

The authors declare that they have no competing interests.

\section{Author details}

'Department of Medicine, McMaster University, Hamilton, ON, Canada.

${ }^{2}$ Joseph Brant Hospital, Burlington, ON, Canada.

Received: 6 January 2020 Accepted: 22 July 2020

Published online: 31 July 2020

\section{References}

1. Allerberger F, Wagner M. Listeriosis: a resurgent foodborne infection. Clin Microbiol Infect. 2010;16:16-23.

2. CDC. National Center for Emerging and Zoonotic Infectious Diseases. National Enteric Disease Surveillance:The Listeria Initiative; 2016. p. 1-2.

3. Swaminathan B, Gerner-Smidt P. The epidemiology of human listeriosis. Microbes Infect. 2007:9:1236-43.

4. Hasan T, Chik W, Chen S, Kok J. Successful treatment of Listeria monocytogenes prosthetic valve endocarditis using rifampicin and benzylpenicillin in combination with valve replacement. JMM Case Rep. 2017:4:e005085

5. García-Granja PE, et al. Infective endocarditis due to Listeria Monocytogenes: a report of 4 patients. Rev Esp Cardiol (Engl Ed). 2016;69:700-2.

6. Heysell SK, Hughes MA. Listeria monocytogenes Endovascular Graft Infection. Open Forum Infect Dis. 2016;3:ofv203.

7. Shoai-Tehrani M, et al. Listeria monocytogenes-associated endovascular infections: a study of 71 consecutive cases. J Inf Secur. 2019;79:322-31.

8. CommisionGL CA. Guidelines on the application of general principles of food hygiene to the control of Listeria monocytogenes in foods; 2009. p. 1-28.
9. Scallan E, Griffin PM, Angulo FJ, Tauxe RV, Hoekstra RM. Foodborne illness acquired in the United States--unspecified agents. Emerging Infect Dis. 2011;17:16-22

10. Del Pozo JL, de la Garza RG, de Rada PD, Ornilla E, Yuste JR. Listeria monocytogenes septic arthritis in a patient treated with mycophenolate mofetil for polyarteritis nodosa: a case report and review of the literature. Int J Infect Dis. 2013;17:e132-3.

11. Charlier C, et al. Listeria monocytogenes-associated joint and bone infections: a study of 43 consecutive cases. Clin Infect Dis. 2012;54:240-8.

12. Schett G, Herak P, Graninger W, Smolen JS, Aringer M. Listeria-associated arthritis in a patient undergoing etanercept therapy: case report and review of the literature. J Clin Microbiol. 2005;43:2537-41.

13. Khan KM, Pao W, Kendler J. Epidural abscess and vertebral osteomyelitis caused by Listeria monocytogenes: case report and literature review. Scand J Infect Dis. 2001:33:714-6.

14. Chirgwin K, Gleich S. Listeria monocytogenes osteomyelitis. Arch Intern Med. 1989;149:931-2.

15. Morrison RE, Brown J, Gooding RS. Spinal cord abscess caused by Listeria monocytogenes. Arch Neurol. 1980;37:243-4.

16. Pfadenhauer K, Rossmanith T. Spinal manifestation of neurolisteriosis. J Neurol. 1995;242:153-6.

17. Aubin $\mathrm{GG}$, et al. Unusual case of spondylodiscitis due to Listeria monocytogenes. J Bone Jt Infect. 2016;1:7-9.

18. Berbari EF, et al. 2015 Infectious Diseases Society of America (IDSA) clinica practice guidelines for the diagnosis and treatment of native vertebral osteomyelitis in adults. Clin Infect Dis. 2015;61:e26-46.

19. Sehn JK, Gilula LA. Percutaneous needle biopsy in diagnosis and identification of causative organisms in cases of suspected vertebral osteomyelitis. Eur J Radiol. 2012;81:940-6.

20. Chew FS, Kline MJ. Diagnostic yield of CT-guided percutaneous aspiration procedures in suspected spontaneous infectious diskitis. Radiology. 2001; 218:211-4.

21. Pupaibool J, Vasoo S, Erwin PJ, Murad MH, Berbari EF. The utility of imageguided percutaneous needle aspiration biopsy for the diagnosis of spontaneous vertebral osteomyelitis: a systematic review and meta-analysis. Spine J. 2015;15:122-31.

22. Kornblum MB, Wesolowski DP. Fischgrund JS, Herkowitz HN. Computed tomography-guided biopsy of the spine. A review of 103 patients. Spine. 1998;23:81-5

23. de Lucas EM, et al. CT-guided fine-needle aspiration in vertebral osteomyelitis: true usefulness of a common practice. Clin Rheumatol. 2009: 28:315-20.

24. McNamara AL, Dickerson EC, Gomez-Hassan DM, Cinti SK, Srinivasan A. Yield of image-guided needle biopsy for infectious Discitis: a systematic review and meta-analysis. AJNR Am J Neuroradiol. 2017;38:2021-7.

25. Trampuz A, et al. Sonication of removed hip and knee prostheses for diagnosis of infection. N Engl J Med. 2007;357:654-63.

26. Marschall J, et al. The impact of prebiopsy antibiotics on pathogen recovery in hematogenous vertebral osteomyelitis. Clin Infect Dis. 2011;52:867-72.

\section{Publisher's Note}

Springer Nature remains neutral with regard to jurisdictional claims in published maps and institutional affiliations.

Ready to submit your research? Choose BMC and benefit from:

- fast, convenient online submission

- thorough peer review by experienced researchers in your field

- rapid publication on acceptance

- support for research data, including large and complex data types

- gold Open Access which fosters wider collaboration and increased citations

- maximum visibility for your research: over $100 \mathrm{M}$ website views per year

At $\mathrm{BMC}$, research is always in progress.

Learn more biomedcentral.com/submissions 